to a high of $94.9 \%$ in 1997-1998 ( $p<0.0001)$. For 2692 infections with documented dates of treatment, the median time from specimen collection to treatment was 21 days (75th percentile: 33 days).

Conclusions This high school STD screening and treatment program achieved high treatment rates for both CT and NG, although the treatment effort required strong commitments of various individuals from the screening program, the administrations in participating schools, and the local health services. Differences in treatment rates per school year reflected the dynamic interplay of these various individuals and organisations as well as the commonly high absentee, truancy, and dropout rates among students in the school district.

\section{3-S4.06 PREDICTORS OF CLINICAL TREATMENT FAILURE AMONG MEN WITH IDIOPATHIC NGU}

doi:10.1136/sextrans-2011-050109.126

${ }^{1} \mathrm{D}$ V Colombara, ${ }^{1} \mathrm{~L}$ E Manhart, ${ }^{1} \mathrm{C}$ M Wetmore, ${ }^{2} \mathrm{M}$. S Lowens, ${ }^{1} \mathrm{~N}$ A Kay, ${ }^{1} \mathrm{P}$ A Totten, ${ }^{2} \mathrm{M}$ R Golden. ${ }^{1}$ University of Washington Seattle, USA; ${ }^{2}$ Public Health-Seattle \& King County, University of Washington, Seattle, USA

Background Up to half of men with nongonoccocal urethritis (NGU) have no known aetiology, yet still receive syndromic treatment. Identifying characteristics associated with clinical treatment failure may aid in determining the aetiology of these cases.

Methods From 1 January 2007 to 31 December 2010, 553 men entered a randomised double-blind treatment trial for NGU at the Public Health Seattle \& King County STD clinic in Washington. Eligible men had visible urethral discharge or $\geq 5 \mathrm{PMNs} /$ high power field on a Gram stained slide of urethral exudates. Men were randomised to either $1 \mathrm{~g}$ single dose azithromycin or $100 \mathrm{mg}$ doxycycline twice daily for 7 days. Chlamydia trachomatis, Neisseria gonorrhoeae, and Trichomonas vaginalis were assessed by TMA (Gen-Probe, Inc., San Diego, CA); Mycoplasma genitalium was assessed by an in-house PCR assay. Ureaplasmas were detected by culture and speciated by a Ureaplasma urealyticum-biovar two specific PCR. Men negative for all pathogens were considered idiopathic and invited to return $2-5$ weeks after enrolment. Clinical treatment failure was defined as visible urethral discharge or $\geq 5$ PMNs. We evaluated baseline demographic and clinical characteristics, self-reported sexual history at enrolment, sexual practices between visits and depression as potential correlates of clinical treatment failure using log binomial regression.

Results Of the 430 (81\%) men with NGU who returned for followup, 202 (47\%) were considered idiopathic at baseline. Enrollees were $68 \%$ white and $27 \%$ black. Age ranged from 19 to 62 . Fifty-one men (25\%) with idiopathic NGU experienced clinical failure. In multivariate analyses, purulent discharge at enrolment more than doubled the risk of failure ( $\mathrm{ARR}=2.5,95 \% \mathrm{CI}: 1.4 \%$ to $4.4 \%$ ) and black men were nearly twice as likely as non-blacks to have treatment failure $(A R R=1.8,1.1$ to 2.8). Age, socioeconomic status, number of partners in last 2 months, sexual orientation, sexual behaviour (anal/ vaginal sex, unprotected sex between visits), depression, and other baseline clinical characteristics were not associated with treatment failure see Abstract O3-S4.06 table 1.

Conclusions Treatment failure was common among men with idiopathic NGU and associated with black race and purulent discharge at enrolment. The association with purulent discharge suggests an etiologic agent that evokes a robust immune response. Insofar as race defines sexual networks, an etiologic agent present in the network may explain the observed differential risk of persistent NGU.
Abstract 03-S4.06 Table 1 Measures of risk for clinical failure among men treated for idiopathic NGU

\begin{tabular}{|c|c|c|c|c|}
\hline \multirow{2}{*}{ Characteristics } & \multicolumn{2}{|l|}{ Univariate analysis } & \multicolumn{2}{|c|}{ Multivariate analysis } \\
\hline & RR (95\% CI) & $\overline{p \text { Value }}$ & RR (95\% CI) & p Value \\
\hline \multicolumn{5}{|l|}{ Demographics } \\
\hline Age & $0.99(0.97$ to 1.02$)$ & 0.591 & & \\
\hline Black race & 1.66 (1.02 to 2.70$)$ & 0.042 & $\begin{array}{l}1.76(1.11 \text { to } \\
2.80)\end{array}$ & 0.016 \\
\hline Low socioeconomic status* & $1.24(0.76$ to 2.02$)$ & 0.394 & & \\
\hline \multicolumn{5}{|l|}{ Baseline signs and symptoms } \\
\hline PMNs $\geq 10$ & $1.40(0.85$ to 2.31$)$ & 0.190 & & \\
\hline Visible discharge & $1.66(0.65$ to 4.21$)$ & 0.286 & & \\
\hline Purulent discharge on exam & $1.75(0.71$ to 4.28$)$ & 0.223 & $\begin{array}{l}2.51(1.44 \text { to } \\
4.35)\end{array}$ & 0.001 \\
\hline Discharge amount on exam & & 0.789 & & \\
\hline Small & Referent & - & & \\
\hline Moderate & $1.18(0.70$ to 1.99$)$ & 0.536 & & \\
\hline Large & $0.87(0.25$ to 3.08$)$ & 0.834 & & \\
\hline Depression (CES-D score $\geq 10$ ) & 1.02 (0.56 to 1.87$)$ & 0.936 & & \\
\hline \multicolumn{5}{|l|}{ Sexual behaviours } \\
\hline Sex with a man in last 12 months & $0.94(0.56$ to 1.59$)$ & 0.819 & & \\
\hline $\begin{array}{l}\text { Any anal insertive partners } \\
\text { in last } 2 \text { months }\end{array}$ & $0.88(0.51$ to 1.53$)$ & 0.654 & & \\
\hline $\begin{array}{l}\text { Any vaginal sex partners } \\
\text { in last } 2 \text { months }\end{array}$ & 0.79 (0.48 to 1.29$)$ & 0.343 & & \\
\hline $\begin{array}{l}\text { Number sex partners in } \\
\text { last } 2 \text { months }\end{array}$ & 1.02 (0.96 to 1.09$)$ & 0.535 & & \\
\hline $\begin{array}{l}\text { Any unprotected sex } \\
\text { between visits }\end{array}$ & 1.06 (0.56 to 1.98$)$ & 0.864 & & \\
\hline
\end{tabular}

${ }^{*}$ As measured by ability to pay full price for the clinic visit vs payment on a sliding scale.

\section{Clinical sciences oral session 5-treatment: Syphilis, Herpes, \& Bacterial Vaginosis 03-S5.01 IMPACT OF AIC316, A NOVEL ANTIVIRAL HELICASE- PRIMASE INHIBITOR, ON GENITAL HSV SHEDDING: RANDOMISED, DOUBLE-BLIND, PLACEBO-CONTROLLED TRIAL}

doi:10.1136/sextrans-2011-050109.127

${ }^{1} \mathrm{~A}$ Wald, ${ }^{2} \mathrm{~S}$ Stoelben, ${ }^{3} \mathrm{~S}$ Tyring, ${ }^{4} \mathrm{~T}$ Warren, ${ }^{5} \mathrm{C}$ Johnston, ${ }^{1} \mathrm{M} \mathrm{L}$ Huang, ${ }^{2} \mathrm{~B}$ Timmler, ${ }^{2} \mathrm{H}$ Ruebsamen-Schaeff, ${ }^{6} \mathrm{~L}$ Corey, ${ }^{2} \mathrm{~A}$ Birkmann. ${ }^{1}$ University of Washington, Seattle, USA; ${ }^{2}$ AiCuris GmbH \& Co. KG Wuppertal, Germany; ${ }^{3}$ University of Texas Health Science Center Houston, USA; ${ }^{4}$ Westover Heights Clinic, Portland, USA; ${ }^{5}$ University of Washington, Seattle, USA; ${ }^{6}$ Fred Hutchinston Cancer Research Center, University of Washington, Seattle, USA

Background Current treatments for HSV infection are imperfect, do not completely abrogate viral shedding or transmission, and do not interrupt HSV-2-HIV interactions. AIC316 is a helicase-primase inhibitor, a new class of anti-HSV compounds that has a distinct mode of action from currently available nucleoside analogues.

Methods We investigated the safety and efficacy of AIC316 in patients with genital HSV-2 infection in a Phase 2, randomised, multicenter, parallel, double-blind, placebo-controlled trial of 4 different doses of AIC316 administered orally for 4 weeks. Participants were randomised 1:1:1:1:1 to one of the following dose groups: $5 \mathrm{mg}$ given once daily (qd), $25 \mathrm{mg}$ qd, $75 \mathrm{mg} \mathrm{qd}, 400 \mathrm{mg}$ given once-a-week, or matching placebo. Participants in the once daily dose groups received a loading dose. During the trial period participants obtained a swab of genital secretions daily and maintained a diary of genital lesions. The primary endpoint was the rate of genital HSV shedding measured by HSV DNA PCR in the AIC316 groups vs placebo. 
Results 156 (105 women and 51 men) healthy, immunocompetent, HSV-2 positive participants with a history of $1-9$ recurrences per year prior to trial entry, or previous suppressive therapy, were randomised by seven US sites between May 2010 and October 2010. 147 completed the trial. Overall, about 9000 swabs for HSV PCR were collected and assayed for HSV DNA by a sensitive and accurate assay that can detect $>150$ copies $/ \mathrm{ml}$. The first results of these assessments will be presented.

Conclusion The trial will provide insight into the antiviral activity of the novel agent AIC316 for genital HSV infections. This trial design presents a robust and efficient method for evaluating antiviral activity of candidate agents for mucocutaneous HSV infections. These initial efficacy and safety results will lead to selecting the dose for further trials with AIC316.

\section{3-S5.02 FREQUENT BREAKTHROUGH GENITAL HSV-2 SHEDDING ON STANDARD AND HIGH DOSE VALACYCLOVIR}

doi:10.1136/sextrans-2011-050109.128

${ }^{1} \mathrm{C}$ Johnston, ${ }^{2} \mathrm{M}$ Saracino, ${ }^{3} \mathrm{~S}$ Kuntz, ${ }^{1} \mathrm{~A}$ Magaret, ${ }^{1} \mathrm{~J}$ T Schiffer, ${ }^{2} \mathrm{~S}$ Selke, ${ }^{1}$ Meeili Huang, ${ }^{4} \mathrm{~L}$ Corey, ${ }^{1} \mathrm{~A}$ Wald. ${ }^{1}$ University of Washington UW, Fred Hutchinson Cancer Research Center, Seattle, USA; ${ }^{2}$ University of Washington, USA; ${ }^{3}$ Laboratory Medicine, USA; ${ }^{4}$ Fred Hutchinson Cancer Research Center FHCRC, University of Washington UW, USA

Background Short, rapidly cleared, subclinical shedding episodes are the predominant form of HSV-2 reactivation in the genital tract. Valacyclovir $500 \mathrm{mg}$ once daily (SD-VAL) reduces the risk of sexual transmission of herpes simplex virus type 2 (HSV-2) by only $48 \%$. We hypothesised that short HSV-2 shedding episodes occur frequently on SD-VAL and that high dose (HD)-VAL could suppress such episodes of genital HSV-2 shedding.

Methods A randomised open-label crossover study using valacyclovir $500 \mathrm{mg}$ daily (SD-VAL) vs valacyclovir $1 \mathrm{gm}$ three times daily (HD-VAL) was conducted in HSV-2 seropositive, HIV seronegative persons with four or more genital herpes recurrences per year or laboratory confirmed primary genital HSV-2 infection in the previous 6 months. Each study arm lasted for 5 weeks, separated by 1 week wash out. Participants obtained genital swabs four times daily, which were assayed for HSV by quantitative PCR. The primary outcome was frequency of genital HSV shedding on each study arm; secondary outcomes included number and duration of HSV-2 shedding episodes and quantity of virus detected.

Results Forty-three participants collected 9981 genital swabs during the study period. 292 (5.8\%) of 5008 swabs had HSV detected during SD-VAL, compared to 164 (3.3\%) of 4973 on HD-VAL (IRR=0.52, $95 \% \mathrm{CI}=0.43 \%$ to $0.63 \%, \mathrm{p}<0.001)$. Episodes were shorter on $\mathrm{HD}$ VAL (median $7 \mathrm{~h}$, compared to $10 \mathrm{~h}$ on SD-VAL, $\mathrm{p}=0.03$ ) and the median maximum copy number was lower on HD-VAL (3.0 log10 copy $/ \mathrm{ml}$ vs $2.5 \log 10$ copies $/ \mathrm{ml}, \mathrm{p}=0.001)$. However, the annual episode rate was the same regardless of dose; there were 55 shedding episodes over 3.89 person-years of follow-up during SD-VAL (14.1 episodes/year) and 65 episodes over 3.93 person-years during HDVAL (16.5 episodes/year, $\mathrm{p}=0.34$ ).

Conclusion Short bursts of genital HSV-2 reactivation persist during SD-VAL and HD-VAL. Compared to SD-VAL, HD-VAL decreased shedding frequency and episode duration but did not alter episode rate. These data may explain why risk of HSV transmission and HSV-specific genital inflammation persist even in the presence of antiviral therapy. More potent therapies are needed to completely suppress HSV-2 reactivation

ClinicalTrials.gov number NCT00362297.

\section{3-S5.03 HIGH-DOSE VALACYCLOVIR DECREASES PLASMA HIV-1 LEVELS MORE THAN STANDARD DOSE ACYCLOVIR IN HIV-1, HSV-2 POSITIVE PERSONS: A RANDOMISED, CROSSOVER TRIAL}

doi:10.1136/sextrans-2011-050109.129

T Perti, J Baeten, C Johnston, K Diem, N Ochbamichael, Meei-Li Huang, S Selke, A Magaret, L Corey, A Wald. University of Washington, Seattle, USA

Background Standard doses of HSV suppressive therapy reduce plasma HIV-1 levels (0.25-0.5 log10 copies/mL) among HIV-1/HSV2 co-infected persons, and modestly slow disease progression. Putative mechanisms for this effect include direct inhibition of HIV1 by acyclovir or indirect reduction by decreasing HSV-associated inflammation. We hypothesised that higher-dose anti-HSV therapy would result in greater reduction in plasma HIV-1 RNA, and that the effect would be mediated by greater suppression of HSV shedding.

Methods 34 participants with HIV-1 and HSV-2 who were not on antiretroviral therapy were enrolled into a randomised, open-label cross-over trial with valacyclovir $1000 \mathrm{mg}$ twice daily or acyclovir $400 \mathrm{mg}$ twice daily for 12 weeks. After a 2 week wash-out, they were crossed over to the alternate treatment arm for 12 weeks. HSV PCR was performed on self-collected genital swabs obtained daily during the first 4 weeks of each treatment period. Plasma HIV-1 RNA was measured weekly throughout the study.

Results Among the 26 participants who completed both arms of the study, the mean age was 44; 21 were men. At entry, mean CD4 count was 525 cells/mm3 (range, 242-1055) and mean plasma HIV1 RNA $3.9 \log 10$ copies/ml (range 1.2-5.5). The mean plasma HIV RNA was $3.86 \log 10$ copies/ml during acyclovir administration compared with $3.57 \log 10$ copies/ml on valacyclovir; a $0.29 \log 10$ copies $/ \mathrm{ml}$ reduction $(\mathrm{p}=0.002)$. One week after initiation of valacyclovir, plasma HIV RNA decreased by a mean of $0.40 \log 10$ copies/ $\mathrm{ml}$. Valacyclovir reduced HIV-1 RNA by $\geq 0.25 \log 10$ copies $/ \mathrm{ml}$ in 14 (54\%) participants, compared with $3(12 \%)$ on acyclovir. Neither the HSV shedding rate $(8.92 \%$ vs $8.98 \%$ of days, $\mathrm{p}=0.94)$, nor the genital lesion rate $(4.3 \%$ vs $1.1 \% ; p=0.18)$ differed on acyclovir vs valacyclovir.

Conclusions High-dose valacyclovir reduces plasma HIV-1 RNA levels more effectively than standard dose acyclovir in HIV-1, HSV-2 seropositive persons not receiving antiretroviral therapy. High dose valacyclovir does not provide more potent suppression of HSV reactivation in HIV-1 infected persons than acyclovir, suggesting that the effect of valacyclovir on HIV-1 RNA may not be mediated via HSV suppression.

\section{3-S5.04 THE POST-TRIAL EFFECT OF PERIODIC PRESUMPTIVE TREATMENT FOR VAGINAL INFECTIONS ON THE INCIDENCE OF BACTERIAL VAGINOSIS AND LACTOBACILLUS COLONISATION}

doi:10.1136/sextrans-2011-050109.130

${ }^{1} \mathrm{~J}$ Balkus, ${ }^{2} \mathrm{~W}$ Jaoko, ${ }^{3} \mathrm{~K}$ Mandaliya, ${ }^{1} \mathrm{~B}$ Richardson, ${ }^{1} \mathrm{~L}$ Masese, ${ }^{2} \mathrm{R}$ Gitau, ${ }^{2} \mathrm{~J}$ Kiarie, ${ }^{4} \mathrm{~J}$ Marrazzo, ${ }^{4} \mathrm{C}$ Farquhar, ${ }^{4} \mathrm{R}$ S McClelland. ${ }^{1}$ University of Washington, Seattle, USA; ${ }^{2}$ University of Nairobi, Kenya; ${ }^{3}$ Coast Provincial General Hospital, USA; ${ }^{4}$ University of Washington, USA

Background Bacterial vaginosis (BV) is a highly prevalent infection that frequently recurs following standard treatment. In a randomised controlled trial (RCT) of oral periodic presumptive treatment (PPT) to reduce vaginal infections among Kenyan women, we observed a decrease in BV and an increase in Lactobacillus colonisation among women randomised to receive $2 \mathrm{~g}$ metronidazole $+150 \mathrm{mg}$ fluconazole monthly for 12 months. After the trial, 